\title{
Correlation Filters for Detection and Localization of Objects in Degraded Images
}

\author{
Erika M. Ramos-Michel and Vitaly Kober \\ Department of Computer Sciences, Division of Applied Physics, CICESE \\ Km 107 Carretera Tijuana-Ensenada, Ensenada, B.C. 22860, México \\ rmichel@cicese.mx, vkober@cicese.mx
}

\begin{abstract}
Several correlation filters are derived to improve pattern recognition of a noisy target embedded into nonoverlapping background, when the input image is degraded with a linear system. With the help of computer simulation we analyze and compare the performance of various correlation based methods for reliable detection and localization of objects in blurred and noisy images.
\end{abstract}

Keywords: Object recognition, correlation filters, degraded image.

\section{Introduction}

In pattern recognition detection and localization of objects in pictures are tasks of extreme importance. Since the pioneering paper by Vanderlugt [1] various correlation filters have been suggested. When a correlator is used the pattern recognition can be done in two steps. First, the detection is carried out by searching the highest correlation peaks at the filter output, and then the coordinates of the peaks are taken as position estimations of targets in the scene image [2-13].

Several criteria were proposed to characterize the filter performance in terms of signal-to-noise ratio, peak sharpness, light efficiency, discrimination capability, etc. [3]. Correlation filters can be designed by maximizing one of these criteria. Many solutions were proposed when the input scene contains a reference object corrupted by additive noise (overlapping model). For instance, the matched spatial filter (MSF) [1] is derived by maximizing the signal-to-noise ratio (SNR). Horner and Gianino [2] suggested the phase-only filter (POF) that maximizes light efficiency. The optimal filter (OF) [13] was proposed to minimize the probability of anomalous errors (false alarms) $[6,9]$. When the input scene contains a reference object embedded into a disjoint background (nonoverlapping model) and additive noise the following correlation filters were obtained: the generalized optimum filter, which is designed to maximize the ratio of the expected value of the squared correlation peak to the average expected value of the output signal energy (POE) $[4,5,7,10]$; the generalized matched filter that maximizes the ratio of the expected value of the squared correlation peak to the average output variance (SNR) $[4,7,10]$.

The mentioned filters were derived for the overlapping and nonoverlapping models. However, these two models do not take into account possible blurring of input scenes. These kinds of degradation frequently appear during image formation 
and recording [14-16]. For this reason, these degradations should be considered in the design process of filters.

The purpose of this paper is to investigate the performance of correlation filters in terms of accuracy of localization and discrimination capability for a modified nonoverlapping model, which takes into account blurring of the input scene. Two schemes of degradation of the input scene are considered; that is, 1) degradation (blurring) is caused by a linear system with a given point spread function (PSF); 2) degradation consists of blurring by a linear system and further corruption with additive white Gaussian noise. For the proposed signal model new correlation filters optimized with respect to pattern recognition measures are derived. With the help of computer simulation we analyze the performance of the obtained and known filters in terms of discrimination capability (DC) and localization distance errors. We assume that the information of degradation is known. In other words, the parameters of blurring and noise can be estimated taking into account the nature of the degradations. Note that although the degradation information is known, no restoration techniques are utilized because the objective is a reliable recognition and localization of a reference object. The presentation is organized as follows. In Section 2, new correlation filters for the modified nonoverlapping model are presented. Computer simulation results are presented and discussed in Section 3. Finally, Section 4 summarizes our conclusions.

\section{Correlation Filters for Pattern Recognition in Blurred and Noisy Images}

Several causes produce blurred images during the process of image formation: optical diffraction, atmospheric turbulence, out of focus, relative motion between the recording medium and the scene, object moving through a fixed background, etc. In our experiments we consider the blurring degradation due to a relative motion of a camera, while an input scene is still. In this case the captured image is completely blurred. Assume that the input image is always corrupted by additive noise (for instance, sensor's noise).

Let us consider that an input scene $s(x)$ contains a target $t\left(x-x_{0}\right)$ having unknown coordinate $x_{0}$ and a nonoverlapping background $b\left(x, x_{0}\right)$. Because of the image formation process, this image is degraded by a uniform PSF and additive noise as follows:

$$
s\left(x, x_{0}\right)=\left[t\left(x-x_{0}\right)+b\left(x, x_{0}\right)\right] * h_{d}(x)+n(x),
$$

where we use the following notations and assumptions.

1. The nonoverlapping background signal $b\left(x, x_{0}\right)$ is regarded as a product of a realization $b(x)$ from a wide-stationary random process and an inverse support function of the target $w(x)$ defined as zero within the target area and unity elsewhere; that is,

$$
b\left(x, x_{0}\right)=b(x) w\left(x-x_{0}\right)
$$


2. The coordinate $x_{0}$ is considered as a random variable with a uniform probability density function.

3. It is assumed that the wide-sense stationary noise and the target location $x_{0}$ are statistically independent of each other.

4. $\mu_{b}$ is the expected value of $b(x)$.

5. $B_{0}(\omega)$ is the power spectral density of $\left(b(x)-\mu_{b}\right)$, and it is calculated as

$$
B_{0}(\omega)=\left|F\left\{\left[b\left(x, x_{0}\right)-\mu_{b}\right] V(x)\right\}\right|^{2},
$$

where $\mathrm{F}\{$.$\} denotes the Fourier transform. This can be done because b(x)$ is considered as an ergodic process [17]. $V(x)$ is a sinusoidal window that is utilized for smoothing the image in order to obtain a better estimation of the power spectral density. Actually, other windows can be used as well for the estimation [18]. However, the used sinusoidal window is separable, that leads to an easy implementation of the estimation.

6. The input scene is completely degraded by blurring. $h_{d}(x)$ is the real impulse response, which is represented with a uniform PSF as

$$
P S F_{d}=\frac{1}{d^{2}}\left[\begin{array}{ccc}
1 & \cdots & 1 \\
\vdots & \ddots & \vdots \\
1 & \cdots & 1
\end{array}\right]_{d \times d}
$$

and $H_{d}(\omega)$ is its transfer function.

7. $n(x)$ is additive white Gaussian noise with the zero mean and the power spectral density $N(\omega)$.

8. $T(\omega)$ y $W(\omega)$ are the Fourier transforms of $t(x)$ and $w(x)$, respectively.

Next, for the modified nonoverlapping model we derive new optimal correlation filters. Actually, these filters are modified versions of the correlation filters: generalized matched filter (GMF), generalized phase-only filter (GPOF), and generalized optimal filter GOF $[4,9,10,11]$, which are optimal with respect to various recognition measures for the nonoverlapping signal model. The proposed filters are denoted as follows: GMF+, GPOF+, and GOF+. For simplicity, through this paper we use the one-dimensional notation.

The GOF+ filter can be obtained by maximizing the POE criterion defined as

$$
P O E=\frac{\left|E\left[y\left(x, x_{0}\right)\right]\right|^{2}}{E\left\{\left[y\left(x, x_{0}\right)\right]^{2}\right\}}=\frac{\left|E\left[y\left(x, x_{0}\right)\right]\right|^{2}}{\overline{\operatorname{Var}\left\{y\left(x, x_{0}\right)\right\}}+\overline{\left|E\left\{y\left(x, x_{0}\right)\right\}\right|^{2}}} .
$$

First, $\left|E\left[y\left(x, x_{0}\right)\right]\right|^{2}, \overline{\operatorname{Var}\left\{y\left(x, x_{0}\right)\right\}}$, and $\overline{\left|E\left\{y\left(x, x_{0}\right)\right\}\right|^{2}}$ are calculated taking into account (1), and then they are substituted in (5) as follows:

$$
P O E=\frac{\frac{1}{4 \pi^{2}}\left|\int\left[T(\omega)+\mu_{b} W(\omega)\right] H_{d}(\omega) H(\omega) d \omega\right|^{2}}{\frac{1}{2 \pi L} \int\left\{\left[\alpha B_{0}(\omega) \circ|W(\omega)|^{2}+\left|T(\omega)+\mu_{b} W(\omega)\right|^{2}\right]\left|H_{d}(\omega)\right|^{2}+N(\omega)\right\}|H(\omega)|^{2} d \omega} .
$$


Finally, applying the Schwarz inequality [19], we have the filter that maximizes the POE criterion:

$$
H_{G O F+}(\omega)=\frac{\left[T^{*}(\omega)+\mu_{b} W^{*}(\omega)\right] H_{d}^{*}(\omega)}{\left[\left|T(\omega)+\mu_{b} W(\omega)\right|^{2}+\alpha B_{0}(\omega) \circ|W(\omega)|^{2}\right]\left|H_{d}(\omega)\right|^{2}+N(\omega)} .
$$

The asterisk denotes the complex conjugate and the symbol "॰" indicates the convolution operation. $\alpha$ is a normalizing constant [10].

In a similar manner, the modified filters GMF+ and GOF+ are derived and given, respectively, by

$$
\begin{gathered}
H_{G M F+}(\omega)=\frac{\left[T^{*}(\omega)+\mu_{b} W^{*}(\omega)\right] H_{d}^{*}(\omega)}{\left[\alpha B_{0}(\omega) \circ|W(\omega)|^{2}\right]\left|H_{d}(\omega)\right|^{2}+N(\omega)}, \\
H_{G P O F+}(\omega)=\frac{\left[T^{*}(\omega)+\mu_{b} W^{*}(\omega)\right]}{\left|T(\omega)+\mu_{b} W(\omega)\right|} e^{-j \theta_{H_{d}}} .
\end{gathered}
$$

If the observed image has no blurring, the classical generalized filters and obtained modified filters are equal. Note that the filters GPOF and GPOF+ do not take into account the additive noise influence. If the PSF is symmetric, its Fourier transform has zero phase.

\section{Discussion and Computer Simulations}

In this section computer simulation results are presented and discussed. In our experiments the target image $t(x)$ and the background image $b(x)$ possess characteristics given in Table 1 . All the correlation filters are implemented with the use of the Discrete Fourier Transform [15].

Table 1. Parameters of the used images

\begin{tabular}{ccc}
\hline Statistical Description & Background Image & Target Image \\
\hline Mean & 133.0900 & 143.9833 \\
Standard Deviation & 22.5315 & 70.23 \\
Size (pixels) & $256 \times 256$ & $41 \times 69$ \\
\hline
\end{tabular}

The signal range is [0-255]. The target is shown in Fig. 1(a). The nonoverlapping background scene is shown in Fig. 1(b). The observed image is degraded with 1) only a linear system possessing the PSF of different sizes $(\mathrm{d})$; 2) a linear system and additive white Gaussian noise (AWGN) with zero mean and the standard deviations $\left(\sigma_{\mathrm{n}}\right): 5,10,15,20,25,30,35,40$ and 43 . Figure 2 shows an example of the observed image, which is degraded by the PSF with $d=5$, and the noise with $\sigma_{n}=20$. 


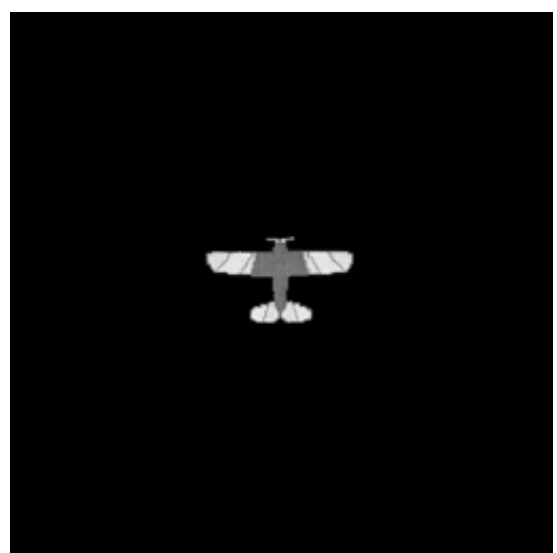

(a)

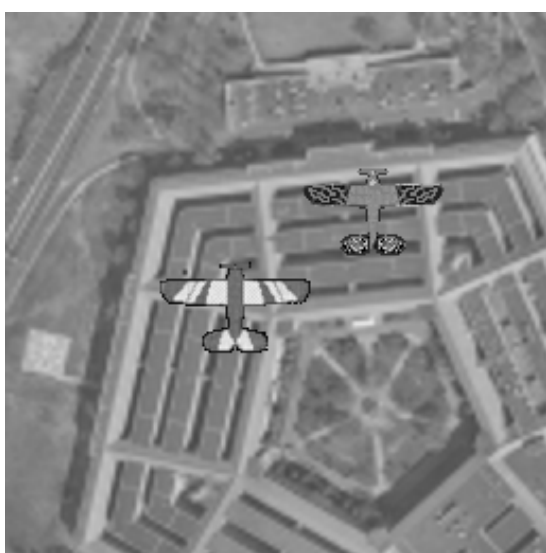

(b)

Fig. 1. (a) Image target, (b) background image

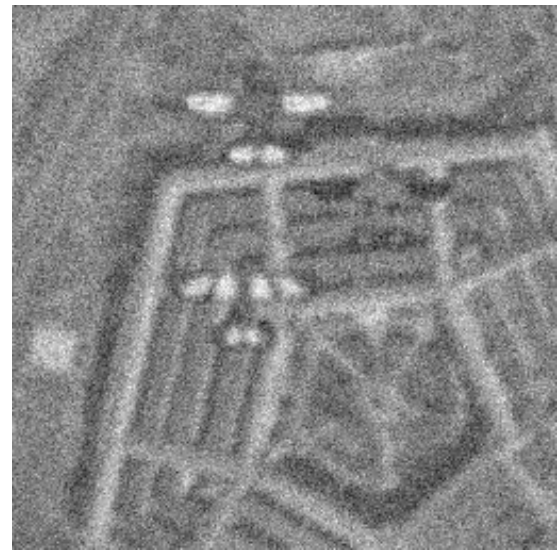

Fig. 2. Observed image with a uniform PSF (size $d=5$ ) and AWGN with $\mu_{\mathrm{n}}=0$ and $\sigma_{n}=20$

The discrimination capability is formally defined as ability of a filter to distinguish a target among other different objects. If a target is embedded into a background that contains false objects, then the DC can be expressed as follows:

$$
D C=1-\frac{\left|C^{B}(0,0)\right|^{2}}{\left|C^{T}(0,0)\right|^{2}},
$$

where $C^{B}$ is the maximum in the correlation plane over the background area to be rejected, and $C^{T}$ is the maximum in the correlation plane over the area of target position. The area of target position is determined in the close vicinity of the actual target location. The background area is complementary to the area of target position. The DC gives a detection ability of a filter. The location errors can be characterized by deviation between actual and obtained target positions. Formally, the distance error 
of localization is calculated as the distance between the known exact position of the target $\left(x_{T}, y_{T}\right)$ and the position corresponding to the coordinates of the maximum value in the correlation plane over the area of object to be recognized was found $\left(\tilde{x}_{T}, \tilde{y}_{T}\right)$.

$$
\mathrm{DE}=\sqrt{\left(x_{T}-\tilde{x}_{T}\right)^{2}+\left(y_{T}-\tilde{y}_{T}\right)^{2}} .
$$

\subsection{Results of Scenario 1}

In this scenario, the observed image is non-degraded. Table 2 shows the performance of the correlation filters in terms of the DC. To obtain these results the 30 statistical trials were carried out. In each trial the target position randomly was changed. We see that all the filters are able to detect and to localize the target. Note that the GOF and the OF filters yield the best results.

Table 2. Mean and Standard Deviation $(S D)$ of $D C$ of Scenario 1

\begin{tabular}{ccccccc}
\hline Statistics & MSF & POF & OF & GMF & GPOF & GOF \\
\hline Mean & 0.1333 & 0.8698 & 0.9284 & 0.3954 & 0.8734 & 0.9395 \\
SD & 0.0078 & 0.0087 & 0.0092 & 0.0080 & 0.0131 & 0.0084 \\
\hline
\end{tabular}

\subsection{Results of Scenario 2}

In this scenario, the observed image is degraded only by a linear system with the uniform PSFs, whose the sizes are 3, 5, 7, 11, and 15. Table 3 shows the performance of the correlation filters in terms of the DC. For each blurring degradation 30 statistical trails were conducted.

Table 3. Mean and Standard Deviation $(S D)$ of $D C$ of Scenario 2

\begin{tabular}{|c|c|c|c|c|c|c|c|c|c|c|}
\hline Size of PSF & \multicolumn{2}{|c|}{3} & \multicolumn{2}{|c|}{5} & \multicolumn{2}{|c|}{7} & \multicolumn{2}{|c|}{11} & \multicolumn{2}{|r|}{15} \\
\hline Filters & Mean & SD & Mean & SD & Mean & SD & Mean & SD & Mean & SD \\
\hline POF & 0.7889 & 0.0192 & 0.6359 & 0.0246 & 0.4505 & 0.0311 & 0.3095 & 0.0297 & 0.1710 & 0.0302 \\
\hline $\boldsymbol{O F}$ & 0.9271 & 0.0076 & 0.9189 & 0.0081 & 0.9172 & 0.0090 & 0.8960 & 0.0173 & 0.9108 & 0.0079 \\
\hline GMF & 0.2945 & 0.0081 & 0.2102 & 0.0085 & 0.1484 & 0.0089 & & & - & \\
\hline GPOF, GPOF+ & 0.7611 & 0.0191 & 0.6973 & 0.0120 & 0.5363 & 0.0185 & 0.3796 & 0.0236 & 0.2484 & 0.0260 \\
\hline$G O F$ & 0.8794 & 0.0152 & 0.8460 & 0.0207 & 0.7682 & 0.0334 & 0.7405 & 0.0346 & 0.6486 & 0.0690 \\
\hline GMF+ & 0.3954 & 0.0080 & 0.3954 & 0.0080 & 0.3954 & 0.0080 & 0.3954 & 0.0080 & 0.3954 & 0.0080 \\
\hline GOF+ & 0.9395 & 0.0084 & 0.9395 & 0.0084 & 0.9395 & 0.0084 & 0.9395 & 0.0084 & 0.9395 & 0.0084 \\
\hline
\end{tabular}

We see that in this case the proposed filters taking into account the blurring parameters yield essentially better performance than those of the classical filters. The modified GOF+ is the best. The MSF filter always fails to recognize the object. The GMF begins to fail when the size of the PSF is greater than 9. Note that if a filter is able to detect the object then this filter localizes exactly the position of the target. 
Because the number of observations is sufficient, it is possible to employ a normal distribution and to calculate a $95 \%$ confidence interval as follows:

$$
P\left(\mu-1.96 \frac{\sigma}{\sqrt{N}} \leq D C \leq \mu+1.96 \frac{\sigma}{\sqrt{N}}\right)=0.95 \text {. }
$$

With $95 \%$ confidence the DC interval of each of the filters can be calculated. For instance, for the GOF+ the confidence intervals for different PSFs are $(0.9366$, $0.9424)$.

\subsection{Results of Scenario 3}

In this scenario, the observed image is degraded both by a linear system with the uniform PSFs, whose sizes are $3,5,7,9,11,15$, and due to additive noise with standard deviations of $5,10,15,20,25,30,35,40$, and 43 .

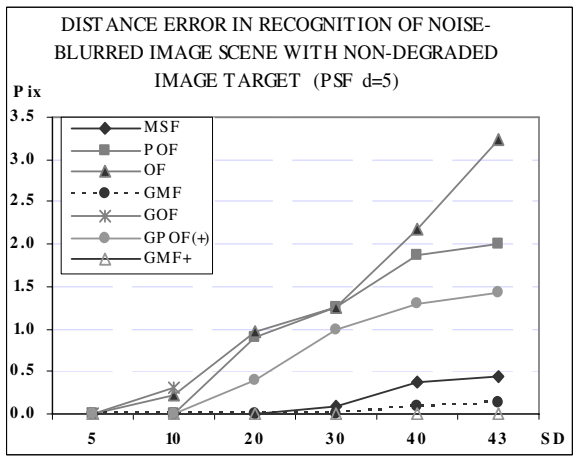

(a)

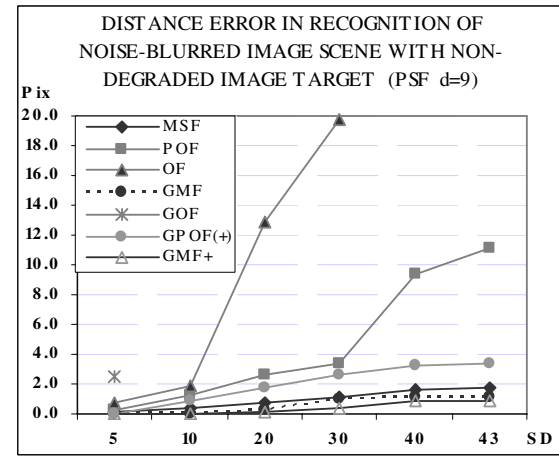

(b)

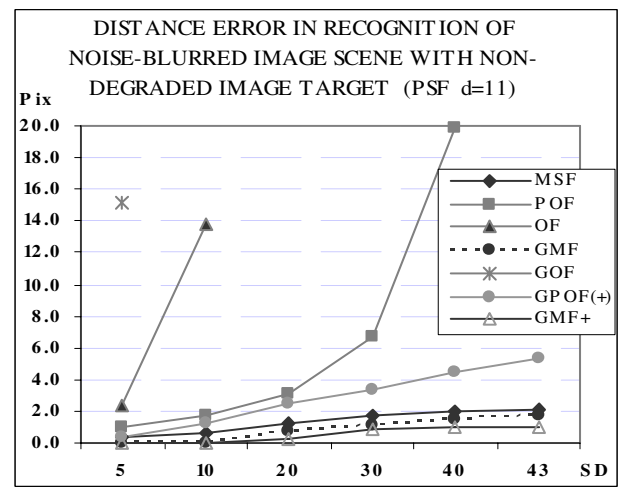

(c)

Fig. 3. Distance errors of localization with the PSF's sizes: (a) $d=5$, (b) $d=9$, (c) $d=11$

In this case the performance of the correlation filters in terms of the DC is similar to that of Scenario 2. However, the distance errors of localization increase, while the standard deviation of the noise increases. Figure 3 shows the performance of the 
filters with respect to the distance errors of localization. It can be seen that the GMF+ yields the best performance in terms of this criterion.

\section{Conclusions}

In this paper three correlation filters were proposed in order to improve detection and localization of a noisy target embedded into nonoverlapping background degraded with a linear system. With the help of computer simulation we showed that the proposed filters taking into account information about degradation yield better results in terms of detection and localization than those of the known correlation filters.

It is necessary to investigate the performance of pattern recognition systems consisting of the two separate steps; that is, the first step is image restoration, and then is object recognition with a correlation filter.

\section{References}

1. VanderLugt A. B., Signal Detection by Complex Filtering. IEEE Trans. Inf. Theory, Vol. 10, (1964) 139-135.

2. Horner J. L. and Gianino P. D., Phase-Only Matched Filtering. Applied Optics, Vol. 23, No. 6, (1984) 812-816.

3. Vijaya-Kumar B. V. K. and Hassebrook L., Performance Measures for Correlation Filters. Applied Optics, Vol. 29, No. 20, (1990) 2997-3006.

4. Javidi B. and Wang J., Design of Filters to Detect a Noisy Target in Nonoverlapping Background Noise. Journal OSA (A), Vol. 11, No. 10, (1994) 2604-2612.

5. Vijaya-Kumar B. V. K., Correlation Filters Minimizing Peak Location Errors. Journal OSA (A), Vol. 9, No. 5, (1992) 678-682.

6. Yaroslavsky L. P., The theory of optimal methods for localization of objects in pictures. In: Progress in Optics XXXII, E. Wolf, Ed., Elsevier, (1993) 145-201.

7. Javidi B. and Horner Joseph L., Real-Time Optical Information Processing. Academic Press, San Diego, California (1994).

8. Ahouzi E., Campos J. and Yzuel, M. J., Phase-Only Filter with Improved Discrimination. Journal OSA (A), Vol. 19, No. 17, (1994) 1340-1342.

9. Javidi B., Parchekani F. and Zhang G., Minimum-Mean-Square-Error Filters for Detecting a Noisy Target in Background Noise. Applied Optics, Vol. 35, (1996) 6964-6975.

10. Kober V. and Campos J., Accuracy of Location Measurement of a Noisy Target in a Nonoverlapping Background. Journal OSA (A), Vol. 13, No. 8, (1996) 1653-1666.

11. Towghi N. and Javidi B., Generalized Optimum Receiver for Pattern Recognition with Multiplicative, Additive, and Nonoverlapping Background Noise. Journal OSA (A), Vol. 15, No. 6, (1998) 1557-1565.

12. Yaroslavsky L. P., Is the Phase-Only Filter and Its Modifications Optimal in Terms of the Discrimination Capability in Pattern Recognition?. Applied Optics, Vol. 31, No. 11, (1998) 1677-1679.

13. Kober, V. I. and Ovseyevich A., Phase-Only Filter with Improved Filter Efficiency and Correlation Discrimination. Pattern Recognition and Image Analysis, Vol. 10, No 4, (2000) 514-519.

14. Jain Anil K., Fundamental of Digital Image Processing. First Edition. Prentice Hall, NJ, (1988). 
15. Biemond J., Lagendijk R. L. and Mersereau R. M., Iterative Methods for Image Deblurring. Proceedings of the IEEE. Vol 78, No. 5, (1990) 856-883.

16. Bertero M. and Boccacci P. Introduction to Inverse Problems in Imaging. Institute of Physics Publising, Bristol and Philadelphia, (1998).

17. Pratt, W. K. Digital Image Processing. Wiley-Interscience Publication. New York, (1981).

18. Jähne, B. Digital Image Processing. Springer. Germany, (2002).

19. Papoulis, A. Probability, Random Variables, and Stochastic Processes. McGraw-Hill, New York, (1984). 